\title{
Modular Enantioselective Synthesis of cis-Cyclopropanes \\ through Redox-Active Carbene Transfer and Stereoselective Photo-Decarboxylation
}

\author{
Matteo Costantini and Abraham Mendoza* \\ Department of Organic Chemistry, Arrhenius Laboratory, Stockholm University, \\ 10691 Stockholm (Sweden)
}

Corresponding author email: abraham.mendoza@su.se

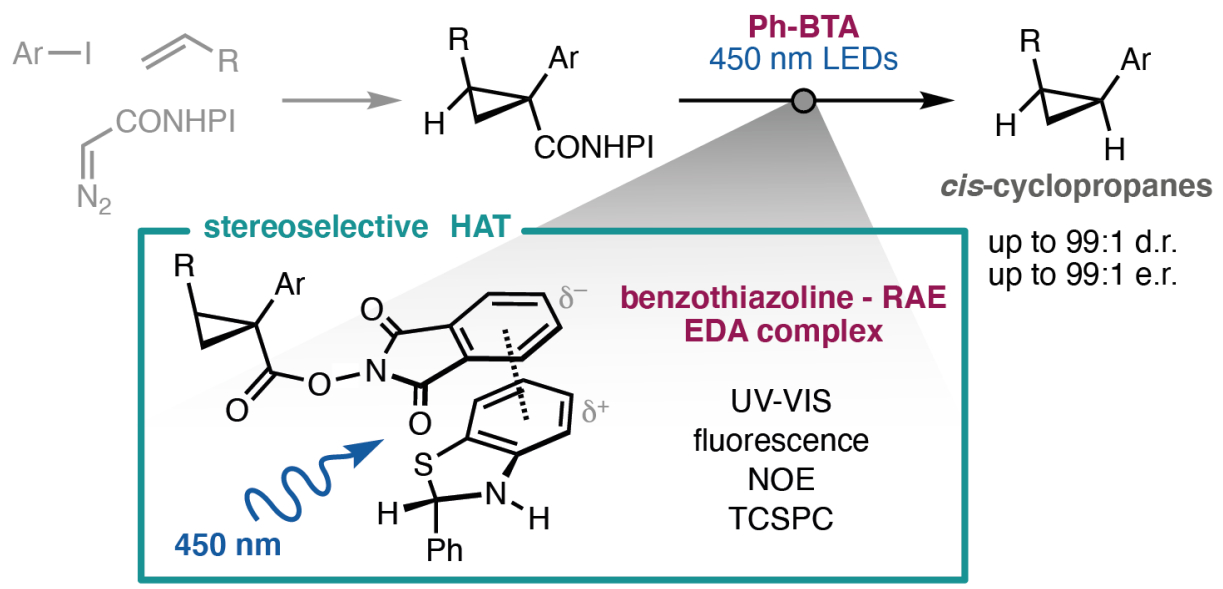

\begin{abstract}
:
Chiral cis-cyclopropanes are strained rigid analogs of alkyl chains, whose study and application is still limited by their difficult synthesis. A modular approach for the synthesis of this challenging structures from abundant olefins is enabled by the discovery of the electron donor-acceptor (EDA) interaction between 2-substituted benzothiazolines and $N$-hydroxyphtalimide esters. These complexes are activated by visible light without photocatalysts. In this system, the benzothiazoline reagent plays a triple role as photoreductant, stereoselective hydrogen atom donor and Brønsted acid. Beyond the enantioselective synthesis of cis-cyclopropanes, these results introduce benzothiazolines as accessible and easily tunable self-sensitized photoreductants.
\end{abstract}




\section{Main text:}

Cyclopropanes are central motifs in organic synthesis. ${ }^{1}$ They have been widely used in the field of medicinal chemistry to improve the properties of potential drug candidates due to their resistance towards metabolic degradation and their structural rigidity (Scheme 1A). ${ }^{1 \mathrm{c}, 2}$ As such, several enantioselective protocols have been developed over the years, mainly targeting the more thermodynamically and kinetically favored trans-cyclopropanes. ${ }^{3}$ In contrast, the synthesis of ciscyclopropanes, an important class of stable and conformationally restricted alkyl chain analogs, ${ }^{1 \mathrm{c}, 2 \mathrm{a}, 4}$ remains a synthetic challenge with only a limited number of protocols reported. ${ }^{5}$

The asymmetric syntheses of these products require the preparation and derivatization of enantiopure $Z$-vinylboronates (Scheme 1B, top-left), ${ }^{6}$ or complex catalytic systems employing transition metals ${ }^{7}$ or engineered proteins. ${ }^{8}$ The more desirable catalytic approaches only offer limited scope ${ }^{9}$ or low diastereo- and enantioselectivity. ${ }^{10}$ In particular, the cis-cyclopropanation of alkenes employing benzylidenes is still problematic, due to the instability of the phenyldiazomethane precursors and the difficult taming of the resulting reactive intermediates. Thus, current methodologies are mostly nonenantioselective, ${ }^{11}$ and the only asymmetric catalytic methods require specific allylic alcohol materials (Scheme 1B, bottom-left). ${ }^{12}$ Seminal studies with chiral iron benzylidene complexes have also been reported, but require stoichiometric chiral complexes and are limited in scope (Scheme 1B, right). ${ }^{13}$ Also, a diastereoselective approach from the chiral pool has been demonstrated in a single example ${ }^{5 f}$ using the decarboxylation of a Barton ester with a large excess of tris(trimethylsilyl)silane to trap the cis-isomer of a cyclopropyl radical intermediate. 


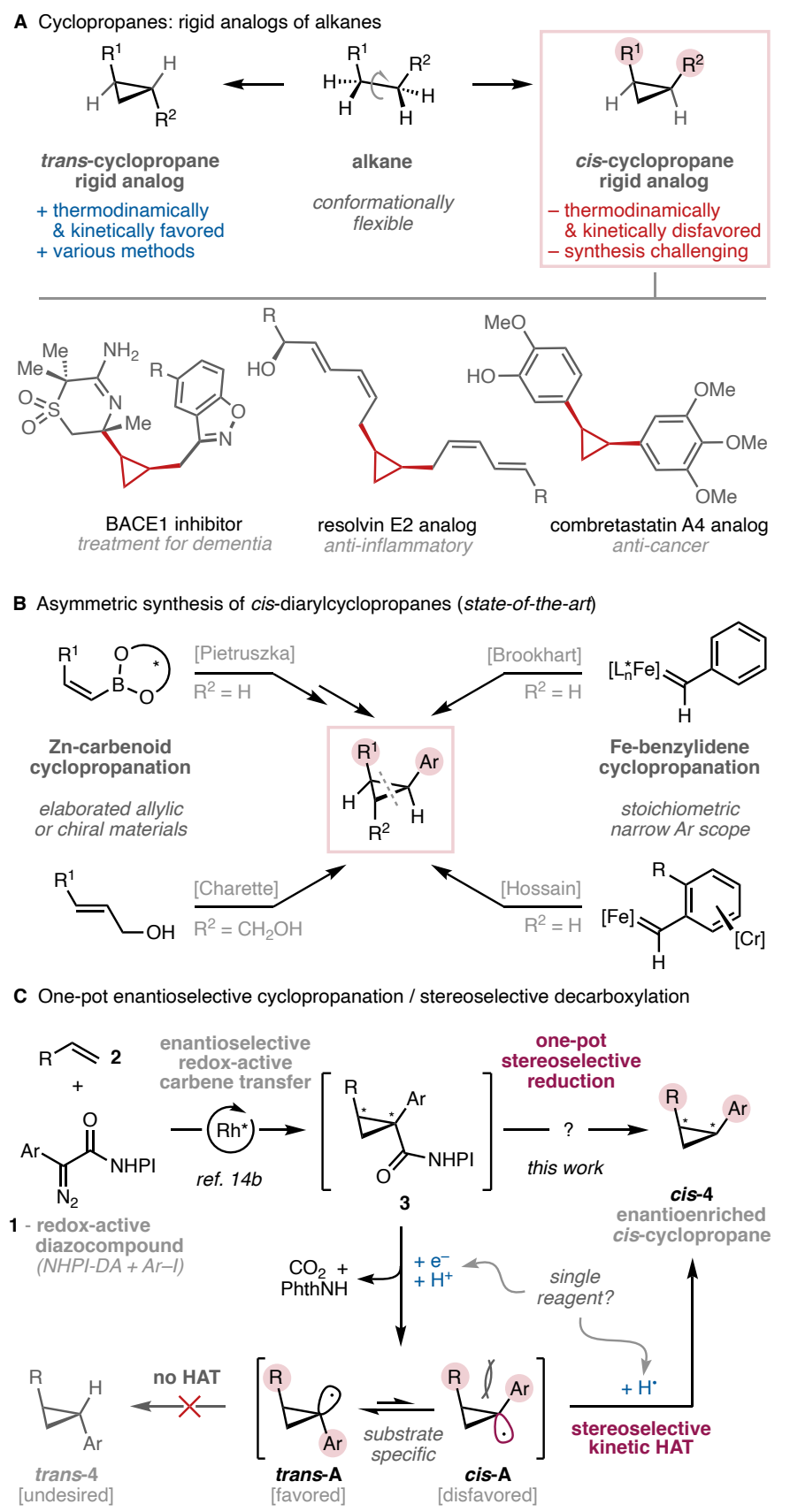

Scheme 1: Current methodologies towards chiral cis-cyclopropanes and our modular approach using redox-active carbenes and stereoselective decarboxylation.

Recently, our group reported the use of redox-active diazoacetate reagents for the general enantioselective synthesis of cyclopropane building blocks from feedstock olefins. ${ }^{14}$ We envisioned that aryl-substituted redox-active diazoacetates $\mathbf{1}$ could be used to convert olefins $\mathbf{2}$ into cisarylcyclopropanes $\mathbf{4}$, by means of sequential asymmetric cyclopropanation and stereoselective decarboxylative reduction of the intermediate cyclopropyl redox-active ester (RAE) 3 (Scheme 1C). Given the higher stability of the trans-pyramidalized radical trans-A, the feasibility of this methodology was contingent upon the design of a suitable hydrogen atom donor that is able to kinetically favor the reduction of the less populated cis-conformer of the cyclopropyl radical cis-A. Ideally, the key HAT reagent should be easily tunable, accessible and autonomous at activating and controlling the stereoselective reduction to deliver a practical and general method. 
Initially, we evaluated known HAT reagents for the reduction of model substrate 3a (Table 1). It was found that the known nickel-catalyzed protocol, ${ }^{15}$ although highly diastereoselective, could only provide the desired cyclopropane cis-4a in low yields (entry 1). In contrast, chloroform ${ }^{16}$ could not afford high stereoselectivity (entry 2). Exploration of the recently discovered photoreduction using $N$-substituted nicotinamides, ${ }^{17}$ and Hantzsch esters, ${ }^{18}$ was promising (entries 3,4), but further attempts to increase the yield or diastereoselectivity by tuning the structure of the dihydropyridines proved unsuccessful (see SI for details). On account of these results, we explored the possibility of employing a reductant with a more sterically hindered hydrogen atom. 2-Substituted benzothiazolines (BTA, 6), have been used as an alternative hydride source to Hantzsch esters in transfer hydrogenation reactions. ${ }^{19}$ More recently, these compounds have been used as hydrogen atom donors in photocatalytic reactions ${ }^{20}$ requiring auxiliary thiyl radical carriers ${ }^{20 \mathrm{~b}}$ or metal photocatalysts. ${ }^{20 \mathrm{a}}$ However, benzothiazolines have never been employed as self-sensitized photoreductants or in reductive decarboxylative reactions as far as we know. A screening of several benzothiazolines (entries 5-10) revealed their unforeseen potential for the desired transformation. In particular, phenyland tert-butyl-benzothiazolines $\mathbf{6 a , b}$ (entries 5,6) provide optimal performance, whereas other substituents provide either lower yields or diastereomeric ratios (entries 7-10). Control experiments with the optimal reagents $\mathbf{6 a , b}$ confirmed the need for blue light irradiation for efficient reduction (entries 11,12). These results introduce the benzothiazoline platform for the design of cheap, easy to handle, readily available and fine-tunable HAT reagents in reductive decarboxylative reactions without any auxiliary light harvesting or chain carrier systems.

Table 1: Optimization of the stereoselective decarboxylative reduction of redox-active ester 3a.

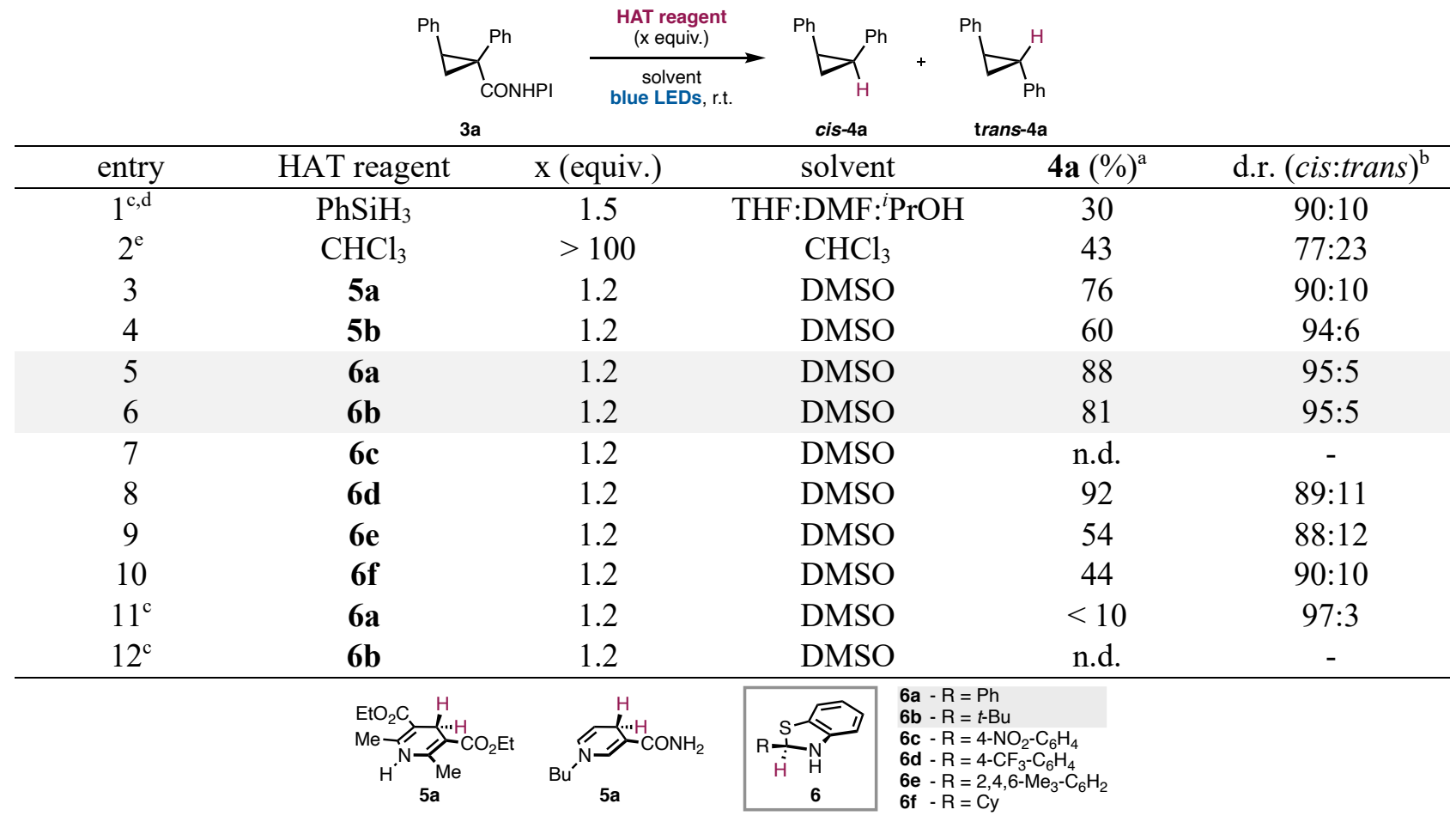

See SI for experimental details. ${ }^{\text {a}}$ Yields measured by ${ }^{1} \mathrm{H}-\mathrm{NMR}$ using 1,1,2,2-tetrachloroethane as an internal standard. ${ }^{b}$ Diastereomeric ratio determined by GC-MS. ${ }^{\mathrm{c}}$ No light irradiation. ${ }^{\mathrm{d}}$ Reaction conditions: $\mathrm{PhSiH}_{3}$ (1.5 equiv.), $\mathrm{Zn}$ (0.5 equiv.), $\mathrm{NiCl}_{2}\left(\mathrm{H}_{2} \mathrm{O}\right)_{6}(10 \mathrm{~mol} \%)$, 4,4'-di- $t$-Bu-2,2'-bipyridyl (20 mol\%), THF:DMF: $\mathrm{PrOH}$ 10:2:1, $40^{\circ} \mathrm{C}$. ${ }^{\mathrm{R}}$ Reaction conditions: $\mathrm{Et}_{3} \mathrm{~N}$ (2 equiv.), 4CzIPN (2 mol\%), $\mathrm{CHCl}_{3}$. 
The simplicity of the new photoreduction system allowed to telescope the cyclopropanation and stereoselective reduction in a one-pot method that deliver cis-cyclopropanes cis-4 from olefins $\mathbf{2}$ and redox-active diazocompounds $\mathbf{1}$. The latter are modularly synthesized from unsubstituted NHPI-DA (7) and aryl iodides 8 through a method previously developed in our group. ${ }^{14 \mathrm{~b}}$ The scope of the onepot synthesis of cis-cyclopropanes was explored using the optimal benzothiazoline 6a, which was easily prepared and stored in multi-gram amounts. For the initial cyclopropanation step, we adapted the recently reported conditions by our group ${ }^{14 \mathrm{~b}}$ using strictly stoichiometric amount of the olefin (1.0 equiv.) and shorter reaction time $(5 \mathrm{~h})$. As shown in Scheme 2A, electron-rich and electron-poor styrenes were tolerated in this transformation, furnishing cis-diarylcyclopropanes $\mathbf{4 b} \mathbf{b}-\mathbf{l}$ in good yields and high enantio- and diastereoselectivities. Substitution in various positions in the aromatic ring were tolerated. Interesting naphthyl (4i) and indolyl (4j) cyclopropanes could also be generated with this protocol. The slightly lower stereoselectivity observed in the tricyclic indene derivative $\mathbf{4 k}$, may be explained by a slower stereo-inversion equilibrium or the particular instability of the corresponding trisubstituted cis-cyclopropyl radical intermediate. Divinyl benzene undergoes double ciscyclopropanation to afford the $C_{2}$-symmetric product $\mathbf{4 l}$ as a single enantiomer in $43 \%$ yield over the four reactions performed in one-pot. It is important to notice that negligible erosion of stereoselectivity was observed for all products relative to the intermediate cyclopropanes, ${ }^{14 \mathrm{~b}}$ indicating that the stereochemical information is conserved throughout the photochemical reduction step. The modular nature of the NHPI-aryldiazoacetates allows for the asymmetric transfer of a variety of aromatic fragments. This way, olefin $\mathbf{2 a}$ can be transformed in a number of ciscyclopropane products decorated with different functionalities (4m-u), that include pendant alkyne (4p), nitrile (4r), and ketone (4t) moieties. To further explore the synthetic potential of this system, we obtained a cis-cyclopropane-modified phenylalanine amino acid (4u) in two steps from commercially available 4-iodophenylalanine. Moreover, the asymmetric total synthesis of the combretastatin A4 analog $4 \mathbf{v}^{6 a}$ was achieved in three steps starting from isovanillin in $39 \%$ overall yield (Scheme 2B). To put this results in perspective, twice as many steps (including a resolution) were previously required to obtain this product in $<10 \%$ overall yield from comparable materials. ${ }^{6 a}$ 
A Scope of the one-pot cis-cyclopropanation of alkenes
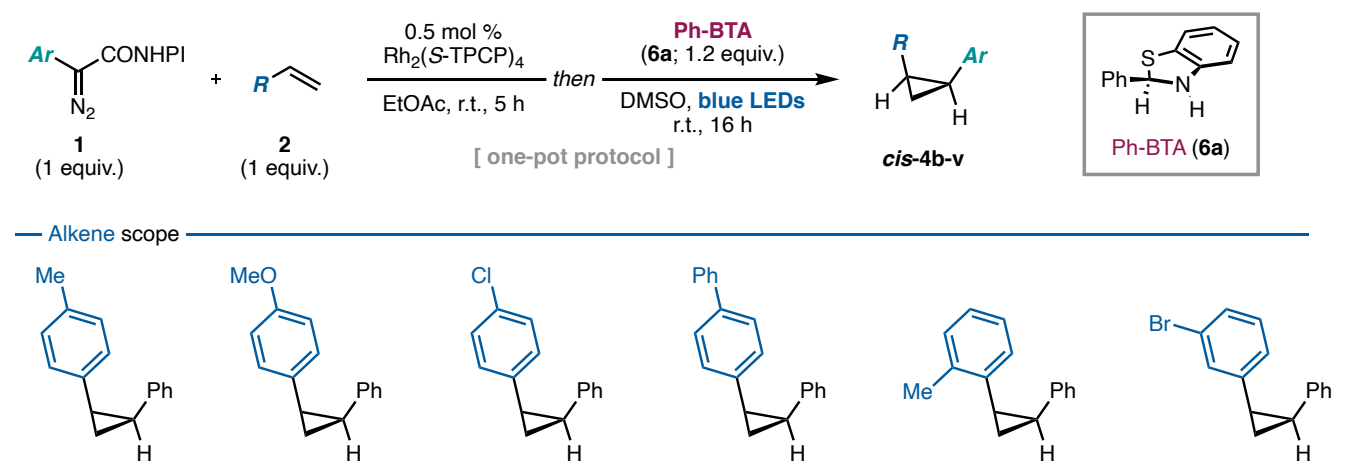

$4 b, 69 \%$

4c, $56 \%$
$96: 4$ d.r., $96: 4$ e.r.

$4 d, 69 \%$

$4 e, 66 \%$
$97: 3$ d.r., $97: 3$ e.r.

4f, $60 \%$
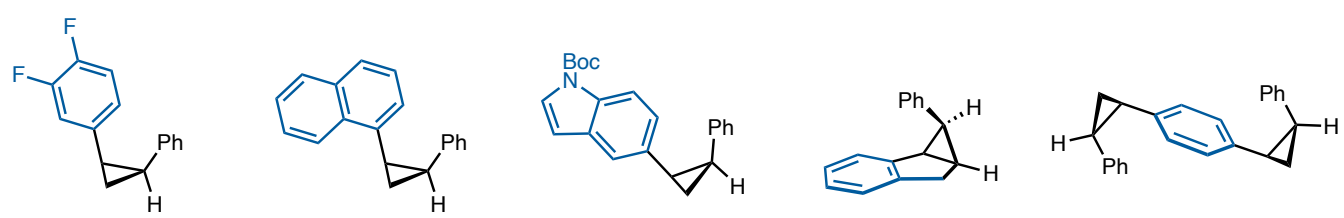

$4 \mathrm{~h}, 73 \%$

$4 i, 59 \%$

$4 \mathbf{j}, 50 \%$

$\mathbf{4 k}, 31 \%$

89:11 d.r., $94: 6$ e.r.

$41,43 \%$

93:7 dr, >99:1 er double cyclopropanation

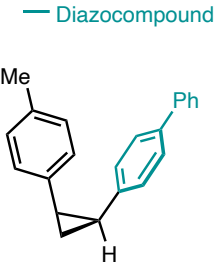

$4 \mathrm{~m}, 42 \%$ 98:2 d.r., $98: 2$ e.r.

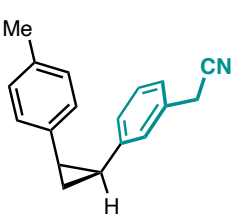

$4 r, 50 \%$

4r, $50 \%$
$95: 5$ d.r., $93: 7$ e.r.

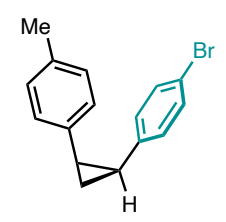

$4 \mathrm{n}, 65 \%$
$97 \cdot 3$.

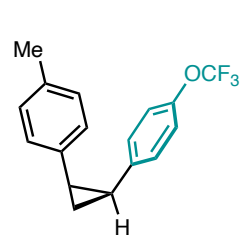

$40,61 \%$

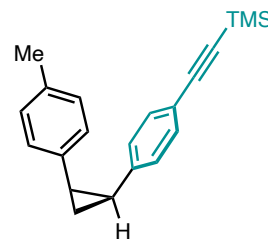

$4 p, 66 \%$ > 99:1 d.r., $96: 4$ e.r.

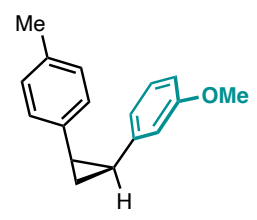

$4 q, 29 \%$ $94: 6$ d.r., $97: 3$ e.r.

B Total synthesis of combrestatin A4 analog
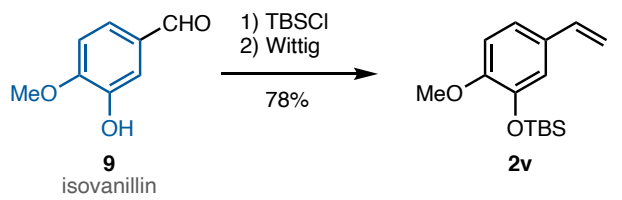

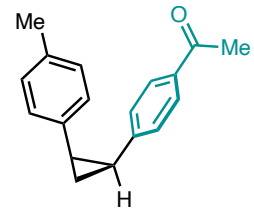

$4 t, 47 \%$ 4 t, $47 \%$
98:2 d.r., $93: 7$ e.r.

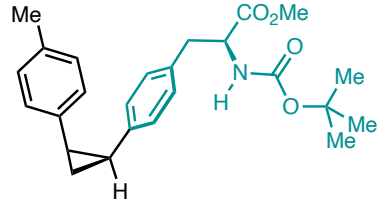

4 u, $25 \%$ cis-cyclopropane 93.7 d.r. aminoacid
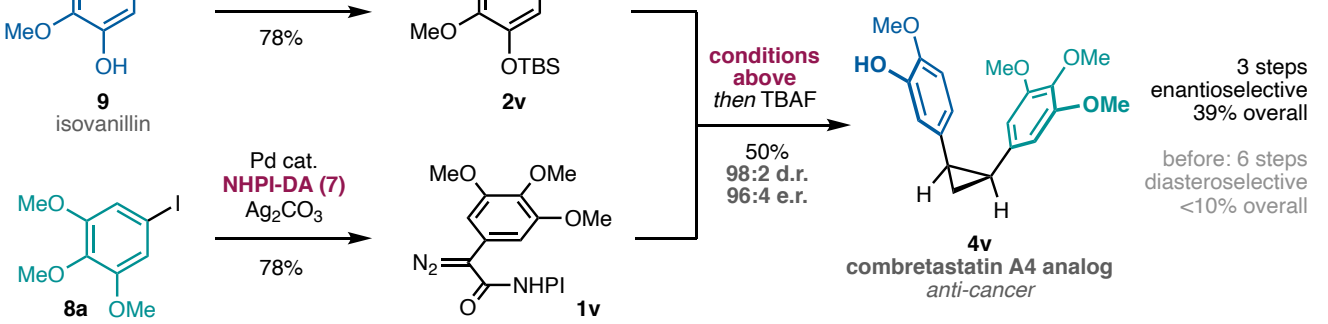

Scheme 2: Scope studies and synthetic applications. Reactions conditions: 1 (1 equiv.), 2 (1 equiv.), $\mathrm{Rh}_{2}(S \text {-TPCP })_{4}(0.5 \mathrm{~mol} \%)$, dry EtOAc $(0.05 \mathrm{M})$, r.t., $5 \mathrm{~h}$; then $6 \mathrm{a}(1.2$ equiv. $)$, dry DMSO $(0.1 \mathrm{M})$, blue LEDs (450 nm), r.t., 16 h. Isolated yields. Diasteromeric ratios determined by HPLC.

The autonomous photoactivation of benzothiazoline 6a was unexpected based on the previously known reactivity of these systems based on HAT-transfer followed by pro-aromatic radical reduction with auxiliary photosensitization or chain carriers. ${ }^{20}$ Thus, photochemical studies were performed to 
investigate the mechanism of the photoreduction. UV-visible spectroscopy revealed that neither 2phenylbenzothiazoline 6a nor NHPI-ester 3a absorb light effectively in the visible range (Figure 1A). Upon mixing, enhanced absorption in the visible range $(450 \mathrm{~nm})$ is observed and a Job plot (Figure 1B) revealed that it is maximum when 3a and 6a are mixed in a 1:1 stoichiometry, consistent with a bimolecular EDA complex ${ }^{21}$ absorbing at the LED irradiation wavelength. Clearly defined excitation and emission features $\left(\lambda_{\max }=435 \mathrm{~nm} ; \lambda_{\mathrm{em}}=490 \mathrm{~nm}\right)$ of the new EDA complex can also be detected by fluorescence (Figure 1C). The formation of this species is further confirmed by time-correlated single photon counting (TCSPC), which allow to identify different fluorescence lifetimes for the benzothiazoline $\mathbf{6 a}\left(\tau_{0}=1.7 \mathrm{~ns}\right)$ and the EDA complex $(\tau=1.4 \mathrm{~ns})$. Stern-Volmer quenching studies performed by increasing the concentration of redox-active ester $\mathbf{3 a}$ revealed an unconventional raise in steady-state fluorescence intensity (see SI), while the corresponding fluorescence lifetime remains constant (Figure 1D). This feature strongly supports a static quenching scenario through the formation of a more emissive bimolecular EDA complex, and it rules out dynamic processes involving the excited state of free benzothiazoline $\left(6 \mathbf{a}^{*}\right)$ that would instead result in a concentrationdependent decrease of the observed fluorescence lifetime.
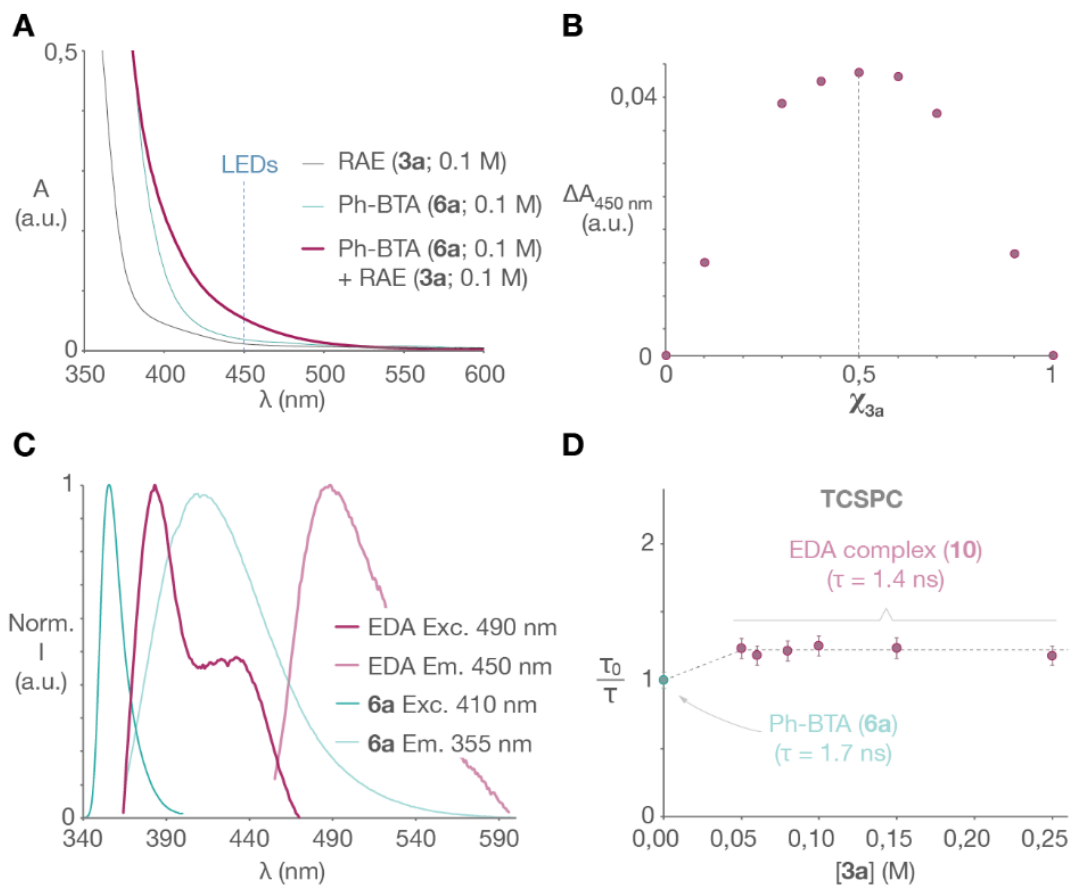

Figure 1: Photophysical characterization of the stereoselective photo-decarboxylation. (A) UVvisible spectrum of NHPI-ester 3a, Ph-BTA (6a) and their 1:1 mixture. (B) Job plot of the mixture between 3a and 6a measured at $450 \mathrm{~nm}\left(\mathrm{c}_{\text {tot }}=0.1 \mathrm{M}\right)$. (C) Normalized excitation and emission spectra of Ph-BTA (6a; $0.02 \mathrm{M})$ and its EDA complex (0.1 M) with NHPI-ester 3a. (D) Lifetime Stern-Volmer plot of Ph-BTA 6a $(\mathrm{c}=0.1 \mathrm{M})$ with NHPI-ester 3a $\left(\lambda_{\mathrm{ex}}=450 \mathrm{~nm}\right)$.

Although we initially hypothesized that the diastereoselectivity would be kinetically controlled by the hydrogen atom transfer (HAT) process, our results could also be explained by a fast stereoretentive HAT before stereo-inversion. To distinguish between these possibilities, the diastereoisomer of the redox-active cyclopropane diast-3a was independently synthesized and subjected to the reaction conditions (Scheme 3A). Similar yield and stereoselectivity for the product cis-4a is 
observed, demonstrating that the stereo-inversion equilibrium is faster than the HAT process, and the latter is kinetically controlled. In principle, benzothiazoline radical cations have two hydrogen atoms susceptible of undergoing the key HAT transfer. To assess their relative contribution, several deuterium incorporation experiments were carried out (Scheme 3B). A first control experiment with DMSO- $\mathrm{d}_{6}$ ruled out any relevant contribution from the solvent. The monodeuterated benzothiazoline at the benzylic carbon 6-d $\mathbf{d}_{1}$ resulted in $70 \%$ deuterium incorporation (56\% yield), while the analogue deuterated in the $\mathrm{N}-\mathrm{H}$ moiety led to $<5 \%$ isotopic labelling and higher efficiency (77\% yield). These observations indicate that the benzylic $\mathrm{C}-\mathrm{H}$ bond is the main hydrogen atom donor, but HAT from either the $\mathrm{N}-\mathrm{H}$ moiety or the imine tautomer ${ }^{22}$ of $\mathbf{6 a}$ may have a secondary role. Indeed, the use of benzothiazoline 6- $\mathbf{d}_{\mathbf{2}}$ increased the degree of deuteration to $>90 \%$, thus accounting for the most relevant HAT processes. These results are consistent with the variable diastereoselectivities observed in the benzothiazoline screening (Table 1) with aliphatic (entries 6,10) and aromatic substituents (entries 5,8,9) of different size in the benzylic position, which affect the relative barriers of the HAT. Furthermore, the quantum yield of the reaction was determined to be $0.09 \pm 0.03$ (Scheme 3C), disfavoring the possibility of a radical-chain mechanism. This behavior contrasts with that of related dihydropyridine systems $\mathbf{5 a}, \mathbf{b}$, operating through radical chain reactions. ${ }^{17 \mathrm{c}, 18 \mathrm{e}}$ The formation of the EDA complex was also directly observed by ${ }^{1} \mathrm{H}-\mathrm{NMR}$ NOE experiments (see SI), ${ }^{23}$ that clearly evidence the spatial proximity of $\mathbf{3 a}$ and $\mathbf{6 a}$ in their equimolar mixture in DMSO (Scheme 3C). 

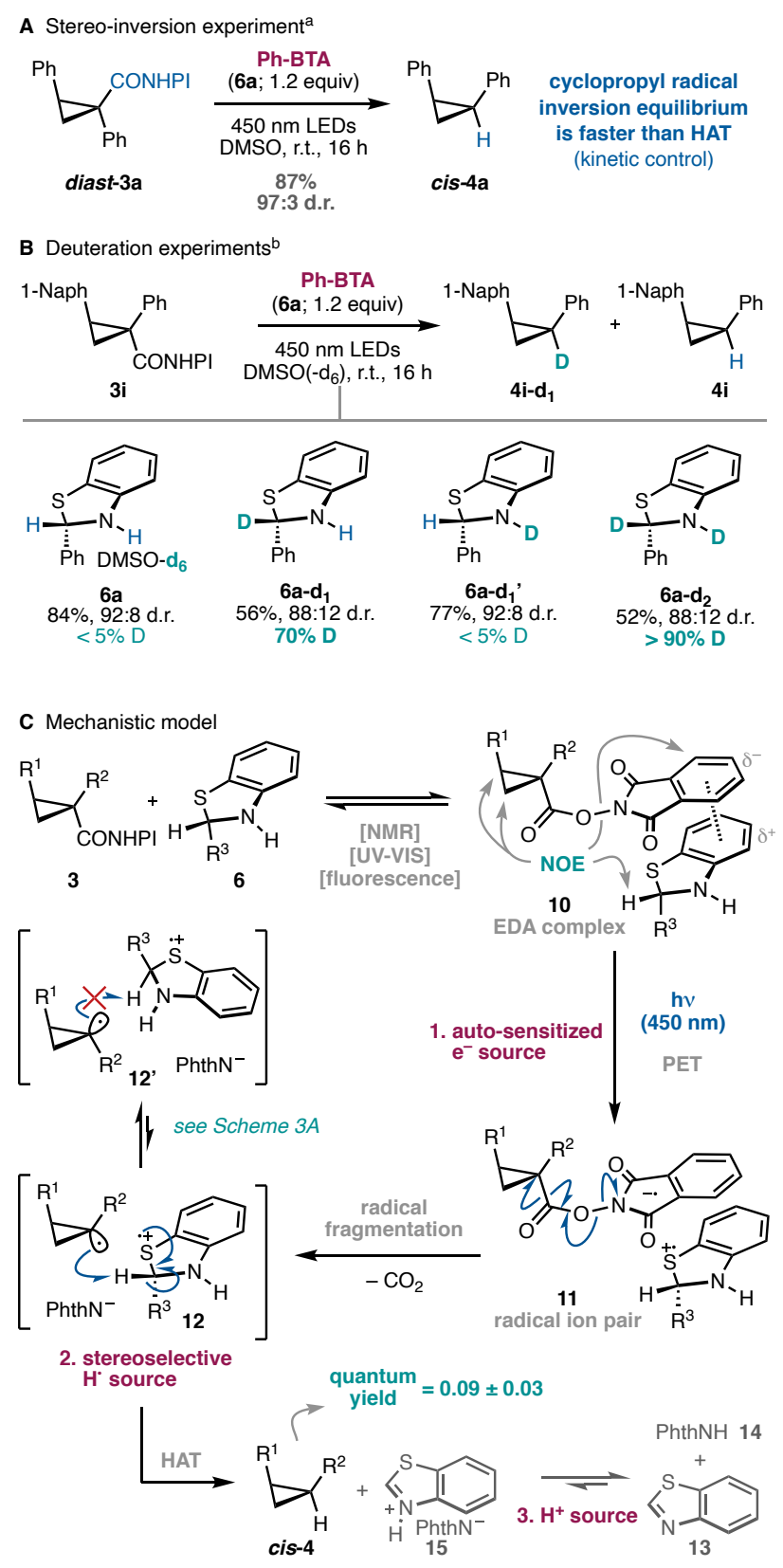

Scheme 3: Mechanistic experiments and model. See SI for details. aDiasteromeric ratios determined

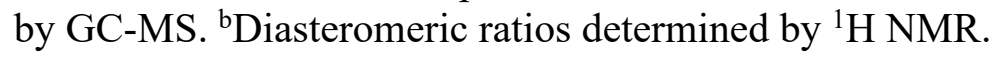

The data presented above supports the mechanism presented in Scheme 3C. Redox-active esters 3 and benzothiazoline 6 associate in solution to form the EDA complex 10, which undergoes photoinduced electron transfer (PET) in the excited state to form the radical ion pair 11. After fragmentation of the NHPI moiety with loss of $\mathrm{CO}_{2}$, the resulting cyclopropyl radical abstracts a hydrogen atom primarily from the benzylic $\mathrm{C}-\mathrm{H}$ bond in the benzothiazoline radical cation (intermediate 12). The alternative HAT process through the $\mathrm{N}-\mathrm{H}$ bond seems to have a secondary role. Either way, the ciscyclopropane product $\mathbf{4}$ is kinetically preferred despite the higher energy of the cis-cyclopropyl radical than the alternative trans-conformer (intermediate 12'). The HAT produces benzothiazole (13) and phtalimide (14) after acid-base reaction of the phthalimidate salt $\mathbf{1 5}$. The alternative possibility of the cyclopropyl radical undergoing HAT directly with the benzothiazoline $\mathbf{6}$ would result in radical chain reactions that can be ruled out based on the quantum yield measurements. Remarkably, the benzothiazoline 6a has a triple role in this system as self-sensitized single-electron photo-reductant 
to promote the fragmentation of the redox-active ester, sterically tuned hydrogen atom source to enhance stereoselectivity, and proton source to neutralize the phthalimidate anion by-product.

In summary, a general and highly enantioselective method to obtain cis-diarylcyclopropanes from olefins and redox-active carbenes has been developed. This protocol allows for quick and modular access to ring- and conformationally-strained compounds from available olefin materials, ultimately facilitating the synthesis of interesting bioactive molecules. These advances are bestowed by a new, efficient and stereoselective photo-decarboxylation driven by a novel EDA complex between redoxactive esters and benzothiazoline reagents. The photophysical properties of the newly discovered system have been investigated, disclosing a new reactivity manifold of benzothiazolines as singleelectron transfer reagents. Beyond enantiopure cis-cyclopropanes, these discoveries open the door for further progress in reductive decarboxylative reactions driven by benzothiazolines as a new platform to develop fine-tuned autonomous photo-reductants.

\section{Acknowledgements:}

We are indebted to the personnel of AstraZeneca Gothenburg and the Dept. of Organic Chemistry at Stockholm University for unrestricted support. The authors are grateful to Ioannis Athanassiadis (ACES, SciLifeLab) for his technical support with GC-HRMS analyses.

\section{Funding:}

Financial support from the Knut and Alice Wallenberg Foundation (KAW2016.0153), and the European Research Council (714737) is gratefully acknowledged.

\section{References:}

1. (a) Chen, D. Y. K.; Pouwer, R. H.; Richard, J.-A., Recent advances in the total synthesis of cyclopropane-containing natural products. Chem. Soc. Rev. 2012, 41 (13), 4631-4642; (b) Ebner, C.; Carreira, E. M., Cyclopropanation Strategies in Recent Total Syntheses. Chem. Rev. 2017, 117 (18), 11651-11679; (c) Talele, T. T., The "Cyclopropyl Fragment" is a Versatile Player that Frequently Appears in Preclinical/Clinical Drug Molecules. J. Med. Chem. 2016, 59 (19), 8712-8756.

2. (a) Martin, S. F.; Dwyer, M. P.; Hartmann, B.; Knight, K. S., Cyclopropane-Derived Peptidomimetics. Design, Synthesis, and Evaluation of Novel Enkephalin Analogues. J. Org. Chem. 2000, 65 (5), 1305-1318; (b) Wipf, P.; Skoda, E. M.; Mann, A., Chapter 11 - Conformational Restriction and Steric Hindrance in Medicinal Chemistry. In The Practice of Medicinal Chemistry (Fourth Edition), Wermuth, C. G.; Aldous, D.; Raboisson, P.; Rognan, D., Eds. Academic Press: San Diego, 2015; pp 279-299.

3. (a) Doyle, M. P.; Forbes, D. C., Recent Advances in Asymmetric Catalytic Metal Carbene Transformations. Chem. Rev. 1998, 98 (2), 911-936; (b) Lebel, H.; Marcoux, J.-F.; Molinaro, C.; Charette, A. B., Stereoselective Cyclopropanation Reactions. Chem. Rev. 2003, 103 (4), 977-1050; (c) Pellissier, H., Recent developments in asymmetric cyclopropanation. Tetrahedron 2008, 64 (30), 7041-7095.

4. (a) Shimamoto, K.; Ohfune, Y., Syntheses and Conformational Analyses of Glutamate Analogs: 2-(2-Carboxy-3-substituted-cyclopropyl)glycines as Useful Probes for Excitatory Amino Acid Receptors. J. Med. Chem. 1996, 39 (2), 407-423; (b) Sekiyama, T.; Hatsuya, S.; Tanaka, Y.; Uchiyama, M.; Ono, N.; Iwayama, S.; Oikawa, M.; Suzuki, K.; Okunishi, M.; Tsuji, T., Synthesis and Antiviral Activity of Novel Acyclic Nucleosides: Discovery of a Cyclopropyl Nucleoside with Potent Inhibitory Activity against Herpesviruses. J. Med. Chem. 1998, 41 (8), 1284-1298; (c) Kazuta, Y.; Hirano, K.; Natsume, K.; Yamada, S.; Kimura, R.; Matsumoto, S.-i.; Furuichi, K.; Matsuda, A.; Shuto, S., Cyclopropane-Based Conformational Restriction of Histamine. (1S,2S)-2-(2-Aminoethyl)-1-(1H-imidazol-4-yl)cyclopropane, a Highly Selective Agonist for the Histamine H3 Receptor, Having a cis-Cyclopropane Structure. J. Med. Chem. 2003, 46 (10), 1980-1988; (d) Watanabe, M.; Kazuta, Y.; Hayashi, H.; Yamada, S.; Matsuda, A.; Shuto, S., Stereochemical Diversity-Oriented Conformational Restriction Strategy. Development of Potent Histamine H3 and/or H4 Receptor Antagonists with an Imidazolylcyclopropane Structure. J. Med. Chem. 2006, 49 (18), 5587-5596; (e) 
Yonezawa, S.; Yamamoto, T.; Yamakawa, H.; Muto, C.; Hosono, M.; Hattori, K.; Higashino, K.; Yutsudo, T.; Iwamoto, H.; Kondo, Y.; Sakagami, M.; Togame, H.; Tanaka, Y.; Nakano, T.; Takemoto, H.; Arisawa, M.; Shuto, S., Conformational Restriction Approach to $\beta$-Secretase (BACE1) Inhibitors: Effect of a Cyclopropane Ring To Induce an Alternative Binding Mode. J. Med. Chem. 2012, 55 (20), 8838-8858.

5. (a) Fang, G.-H.; Yan, Z.-J.; Deng, M.-Z., Palladium-Catalyzed Cross-Coupling of Stereospecific Potassium Cyclopropyl Trifluoroborates with Aryl Bromides. Org. Lett. 2004, 6 (3), 357-360; (b) Piou, T.; Romanov-Michailidis, F.; Ashley, M. A.; Romanova-Michaelides, M.; Rovis, T., Stereodivergent Rhodium(III)-Catalyzed cis-Cyclopropanation Enabled by Multivariate Optimization. J. Am. Chem. Soc. 2018, 140 (30), 9587-9593; (c) Rosenberg, M. L.; Vlašaná, K.; Gupta, N. S.; Wragg, D.; Tilset, M., Highly cisSelective Rh(I)-Catalyzed Cyclopropanation Reactions. J. Org. Chem. 2011, 76 (8), 2465-2470; (d) Spencer, J. A.; Jamieson, C.; Talbot, E. P. A., One-Pot, Three-Step Synthesis of Cyclopropylboronic Acid Pinacol Esters from Synthetically Tractable Propargylic Silyl Ethers. Org. Lett. 2017, 19 (14), 3891-3894; (e) Sugawara, M.; Yoshida, J.-i., Remarkable $\gamma$-Effect of Tin: Acid-Promoted Cyclopropanation Reactions of $\alpha$ ((Alkoxycarbonyl)oxy)stannanes with Alkenes. J. Am. Chem. Soc. 1997, 119 (49), 11986-11987; (f) Yamaguchi, K.; Kazuta, Y.; Abe, H.; Matsuda, A.; Shuto, S., Construction of a cis-Cyclopropane via Reductive Radical Decarboxylation. Enantioselective Synthesis of cis- and trans-1-Arylpiperazyl-2-phenylcyclopropanes Designed as Antidopaminergic Agents. J. Org. Chem. 2003, 68 (24), 9255-9262.

6. (a) Ty, N.; Pontikis, R.; Chabot, G. G.; Devillers, E.; Quentin, L.; Bourg, S.; Florent, J.-C., Synthesis and biological evaluation of enantiomerically pure cyclopropyl analogues of combretastatin A4. Bioorg. Med. Chem. 2013, 21 (5), 1357-1366; (b) Luithle, Joachim E. A.; Pietruszka, J., Synthesis of Enantiomerically Pure cis-Cyclopropylboronic Esters. Eur. J. Org. Chem. 2000, 2000 (14), 2557-2562.

7. (a) Niimi, T.; Uchida, T.; Irie, R.; Katsuki, T., Co(II)-salen-catalyzed highly cis- and enantioselective cyclopropanation. Tetrahedron Lett. 2000, 41 (19), 3647-3651; (b) Suematsu, H.; Kanchiku, S.; Uchida, T.; Katsuki, T., Construction of Aryliridium-Salen Complexes: Enantio- and Cis-Selective Cyclopropanation of Conjugated and Nonconjugated Olefins. J. Am. Chem. Soc. 2008, 130 (31), 10327-10337; (c) Uchida, T.; Irie, R.; Katsuki, T., Cis- and Enantio-selective Cyclopropanation with Chiral $\left(\mathrm{ON}^{+}\right) \mathrm{Ru}-\mathrm{Salen}$ Complex as a Catalyst. Tetrahedron 2000, 56 (22), 3501-3509; (d) Zhu, S.; Perman, J. A.; Zhang, X. P., Acceptor/Acceptor-Substituted Diazo Reagents for Carbene Transfers: Cobalt-Catalyzed Asymmetric ZCyclopropanation of Alkenes with $\alpha$-Nitrodiazoacetates. Angew. Chem. Int. Ed. 2008, 47 (44), 8460-8463.

8. $\quad$ Knight, A. M.; Kan, S. B. J.; Lewis, R. D.; Brandenberg, O. F.; Chen, K.; Arnold, F. H., Diverse Engineered Heme Proteins Enable Stereodivergent Cyclopropanation of Unactivated Alkenes. ACS Cent. Sci. 2018, 4 (3), 372-377.

9. (a) Bachmann, S.; Furler, M.; Mezzetti, A., Cis-Selective Asymmetric Cyclopropanation of Olefins Catalyzed by Five-Coordinate $[\mathrm{RuCl}(\mathrm{PNNP})]^{+}$Complexes. Organometallics 2001, 20 (10), $2102-$ 2108; (b) Bonaccorsi, C.; Mezzetti, A., Optimization or Breakthrough? The First Highly cis- and Enantioselective Asymmetric Cyclopropanation of 1-Octene by "Electronic and Counterion" Tuning of $[\mathrm{RuCl}(\mathrm{PNNP})]^{+}$Catalysts. Organometallics 2005, 24 (21), 4953-4960.

10. (a) Alexander, K.; Cook, S.; Gibson, C. L., cis-Selective cyclopropanations using chiral 5,5diaryl bis(oxazoline) catalysts. Tetrahedron Lett. 2000, 41 (36), 7135-7138; (b) Hu, W.; Timmons, D. J.; Doyle, M. P., In Search of High Stereocontrol for the Construction of cis-Disubstituted Cyclopropane Compounds. Total Synthesis of a Cyclopropane-Configured Urea-PETT Analogue That Is a HIV-1 Reverse Transcriptase Inhibitor. Org. Lett. 2002, 4 (6), 901-904.

11. (a) Verdecchia, M.; Tubaro, C.; Biffis, A., Olefin cyclopropanation with aryl diazocompounds upon catalysis by a dirhodium(II) complex. Tetrahedron Lett. 2011, 52 (10), 1136-1139; (b) Solorio-Alvarado, C. R.; Wang, Y.; Echavarren, A. M., Cyclopropanation with Gold(I) Carbenes by Retro-Buchner Reaction from Cycloheptatrienes. J. Am. Chem. Soc. 2011, 133 (31), 11952-11955; (c) Solorio-Alvarado, C. R.; Echavarren, A. M., Gold-Catalyzed Annulation/Fragmentation: Formation of Free Gold Carbenes by RetroCyclopropanation. J. Am. Chem. Soc. 2010, 132 (34), 11881-11883; (d) Ringger, D. H.; Chen, P., Rational Design of a Gold Carbene Precursor Complex for a Catalytic Cyclopropanation Reaction. Angew. Chem. Int. Ed. 2013, 52 (17), 4686-4689; (e) Lévesque, É.; Goudreau, S. R.; Charette, A. B., Improved Zinc-Catalyzed Simmons-Smith Reaction: Access to Various 1,2,3-Trisubstituted Cyclopropanes. Org. Lett. 2014, 16 (5), 1490-1493; (f) Carden, R. G.; Widenhoefer, R. A., Gold Sulfonium Benzylide Complexes Undergo Efficient Benzylidene Transfer to Alkenes. Chem. Eur. J. 2019, 25 (47), 11026-11030; (g) Aggarwal, V. K.; de Vicente, J.; Bonnert, R. V., Catalytic Cyclopropanation of Alkenes Using Diazo Compounds Generated in Situ. A Novel Route to 2-Arylcyclopropylamines. Org. Lett. 2001, 3 (17), 2785-2788. 
12. Goudreau, S. R.; Charette, A. B., In Situ Generation of Zinc Carbenoids from Diazo Compounds and Zinc Salts: Asymmetric Synthesis of 1,2,3-Substituted Cyclopropanes. J. Am. Chem. Soc. 2009, 131 (43), 15633-15635.

13. (a) Wang, Q.; Mayer, M. F.; Brennan, C.; Yang, F.; Hossain, M. M.; Grubisha, D. S.; Bennett, D., A New Approach to Diastereoselective and Enantioselective Cyclopropane Syntheses Using the Chiral Iron Carbene Complexes $S$ - and $R-\left[\left(\eta^{5}-\mathrm{C}_{5} \mathrm{H}_{5}\right)(\mathrm{CO})_{2} \mathrm{FeCH}\left[\left(\eta^{6}-\mathrm{o}_{-}-\mathrm{CH}_{3} \mathrm{OC}_{6} \mathrm{H}_{4}\right) \mathrm{Cr}(\mathrm{CO})_{3}\right]\right]^{+}$. Tetrahedron 2000, 56 (28), 4881-4891; (b) Wang, Q.; Försterling, F. H.; Hossain, M. M., Enantiospecific Cis-Cyclopropane Synthesis Using the Chiral Iron Carbene Complexes $S$ - and $R-\left(\eta^{5}-\mathrm{C}_{5} \mathrm{H}_{5}\right)(\mathrm{CO})_{2} \mathrm{FeCH}\left[\left(\eta^{6}-\mathrm{o}-\right.\right.$ $\left.\left.\mathrm{CH}_{3} \mathrm{C}_{6} \mathrm{H}_{4}\right) \mathrm{Cr}(\mathrm{CO})_{3}\right]^{+}$. Organometallics 2002, 21 (13), 2596-2598; (c) Theys, R. D.; Hossain, M. M., Asymmetric cyclopropanation reactions via iron carbene complexes having chirality at the carbene ligand. Tetrahedron Lett. 1995, 36 (29), 5113-5116; (d) Seitz, W. J.; Hossain, M. M., Iron Lewis acid catalyzed reactions of phenyldiazomethane and olefins: Formation of cyclopropanes with very high cis selectivity. Tetrahedron Lett. 1994, 35 (41), 7561-7564; (e) Brookhart, M.; Liu, Y.; Goldman, E. W.; Timmers, D. A.; Williams, G. D., Enantioselective cyclopropane syntheses using the chiral carbene complexes $\left(S_{\mathrm{Fe}}\right)-$ and $\left(R_{\mathrm{Fe}}\right)-$ $\mathrm{C}_{5} \mathrm{H}_{5}(\mathrm{CO})\left(\mathrm{PR}_{3}\right) \mathrm{Fe}: \mathrm{CHCH}_{3}{ }^{+}$. A mechanistic analysis of the carbene transfer reaction. J. Am. Chem. Soc. 1991, 113 (3), 927-939; (f) Brookhart, M.; C.Buck, R., Enantioselective benzylidene transfer reactions using the chiral-at-iron benzylidene complexes $\left(S_{\mathrm{Fe}} S_{\mathrm{c}}\right)-$ and $\left(R_{\mathrm{Fe}} S_{\mathrm{c}}\right)-\mathrm{Cp}(\mathrm{CO})\left(\mathrm{Ph}_{2} \mathrm{R}^{\star} \mathrm{P} \mathrm{Fe}_{-} \mathrm{CH}_{6} \mathrm{H}_{5}^{+}\left(\mathrm{R}^{\star}=(S-2-\right.\right.$ methylbutyl) and $S_{\mathrm{Fe}}$ and $\left(R_{\mathrm{Fe}}\right)-\mathrm{Cp}(\mathrm{CO}) \mathrm{PEt}_{3} \mathrm{Fe}=\mathrm{CHC}_{6} \mathrm{H}_{5}^{+}$. J. Organomet. Chem. 1989, 370 (1), 111-127.

14. (a) Montesinos-Magraner, M.; Costantini, M.; Ramírez-Contreras, R.; Muratore, M. E.; Johansson, M. J.; Mendoza, A., General Cyclopropane Assembly by Enantioselective Transfer of a RedoxActive Carbene to Aliphatic Olefins. Angew. Chem. Int. Ed. 2019, 58 (18), 5930-5935; (b) Yu, Z.; Mendoza, A., Enantioselective Assembly of Congested Cyclopropanes using Redox-Active Aryldiazoacetates. ACS Catal. 2019, 9 (9), 7870-7875.

15. Qin, T.; Malins, L. R.; Edwards, J. T.; Merchant, R. R.; Novak, A. J. E.; Zhong, J. Z.; Mills, R. B.; Yan, M.; Yuan, C.; Eastgate, M. D.; Baran, P. S., Nickel-Catalyzed Barton Decarboxylation and Giese Reactions: A Practical Take on Classic Transforms. Angew. Chem. Int. Ed. 2017, 56 (1), 260-265.

16. Ko, E. J.; Savage, G. P.; Williams, C. M.; Tsanaktsidis, J., Reducing the Cost, Smell, and Toxicity of the Barton Reductive Decarboxylation: Chloroform as the Hydrogen Atom Source. Org. Lett. 2011, 13 (8), 1944-1947.

17. $\quad$ (a) Chen, X.; Luo, X.; Peng, X.; Guo, J.; Zai, J.; Wang, P., Catalyst-Free Decarboxylation of Carboxylic Acids and Deoxygenation of Alcohols by Electro-Induced Radical Formation. Chem. Eur. J. 2020, 26, 3226; (b) Zheng, C.; Wang, G.-Z.; Shang, R., Catalyst-free Decarboxylation and Decarboxylative Giese Additions of Alkyl Carboxylates through Photoactivation of Electron Donor-Acceptor Complex. Adv. Synth. Catal. 2019, 361 (19), 4500-4505; (c) Chowdhury, R.; Yu, Z.; Tong, M. L.; Kohlhepp, S. V.; Yin, X.; Mendoza, A., Decarboxylative Alkyl Coupling Promoted by NADH and Blue Light. J. Am. Chem. Soc. 2020, 142 (47), 20143-20151.

18. (a) Buzzetti, L.; Prieto, A.; Roy, S. R.; Melchiorre, P., Radical-Based C-C Bond-Forming Processes Enabled by the Photoexcitation of 4-Alkyl-1,4-dihydropyridines. Angew. Chem. Int. Ed. 2017, 56 (47), 15039-15043; (b) Huang, W.; Cheng, X., Hantzsch Esters as Multifunctional Reagents in Visible-Light Photoredox Catalysis. Synlett 2017, 28 (02), 148-158; (c) Milligan, J. A.; Phelan, J. P.; Badir, S. O.; Molander, G. A., Alkyl Carbon-Carbon Bond Formation by Nickel/Photoredox Cross-Coupling. Angew. Chem. Int. Ed. 2019, 58 (19), 6152-6163; (d) van Leeuwen, T.; Buzzetti, L.; Perego, L. A.; Melchiorre, P., A Redox-Active Nickel Complex that Acts as an Electron Mediator in Photochemical Giese Reactions. Angew. Chem. Int. Ed. 2019, 58 (15), 4953-4957; (e) Wang, P.-Z.; Chen, J.-R.; Xiao, W.-J., Hantzsch esters: an emerging versatile class of reagents in photoredox catalyzed organic synthesis. Org. Biomol. Chem. 2019, 17 (29), 6936-6951.

19. (a) Chikashita, H.; Miyazaki, M.; Itoh, K., 2-Phenylbenzothiazoline as a Reducing Agent in the Conjugate Reduction of $\alpha, \beta$-Unsaturated Carbonyl Compounds. Synthesis 1984, 1984 (04), 308-310; (b) Chikashita, H.; Miyazaki, M.; Itoh, K., Lewis acid-promoted conjugate reduction of $\alpha, \beta$-unsaturated carbonyl compounds by 2-phenylbenzothiazoline (2-phenyl-2,3-dihydrobenzothiazole). J. Chem. Soc., Perkin Trans. 1 1987, (0), 699-706; (c) Enders, D.; Liebich, J. X.; Raabe, G., Organocatalytic Asymmetric Synthesis of trans1,3-Disubstituted Tetrahydroisoquinolines via a Reductive Amination/Aza-Michael Sequence. Chem. Eur. J. 2010, 16 (32), 9763-9766; (d) Henseler, A.; Kato, M.; Mori, K.; Akiyama, T., Chiral Phosphoric Acid Catalyzed Transfer Hydrogenation: Facile Synthetic Access to Highly Optically Active Trifluoromethylated Amines. Angew. Chem. Int. Ed. 2011, 50 (35), 8180-8183; (e) Saito, K.; Akiyama, T., Enantioselective organocatalytic reductive amination of aliphatic ketones by benzothiazoline as hydrogen donor. Chem. Commun. 2012, 48 (38), 4573-4575; (f) Zhu, C.; Akiyama, T., Benzothiazoline: Highly Efficient Reducing 
Agent for the Enantioselective Organocatalytic Transfer Hydrogenation of Ketimines. Org. Lett. 2009, 11 (18), 4180-4183; (g) Zhu, C.; Saito, K.; Yamanaka, M.; Akiyama, T., Benzothiazoline: Versatile Hydrogen Donor for Organocatalytic Transfer Hydrogenation. Acc. Chem. Res. 2015, 48 (2), 388-398.

20. (a) Tarantino, K. T.; Liu, P.; Knowles, R. R., Catalytic Ketyl-Olefin Cyclizations Enabled by Proton-Coupled Electron Transfer. J. Am. Chem. Soc. 2013, 135 (27), 10022-10025; (b) Chen, J.; Huang, W.; Li, Y.; Cheng, X., Visible-Light-Induced Difluoropropargylation Reaction with Benzothiazoline as a Reductant. Adv. Synth. Catal. 2018, 360 (7), 1466-1472; (c) Bhunia, A.; Studer, A., Recent advances in radical chemistry proceeding through pro-aromatic radicals. Chem 2021, DOI: 10.1016/j.chempr.2021.03.023.

21. (a) Lima, C. G. S.; de M. Lima, T.; Duarte, M.; Jurberg, I. D.; Paixão, M. W., Organic Synthesis Enabled by Light-Irradiation of EDA Complexes: Theoretical Background and Synthetic Applications. ACS Catal. 2016, 6 (3), 1389-1407; (b) Mori, T.; Inoue, Y., Charge-transfer excitation: unconventional yet practical means for controlling stereoselectivity in asymmetric photoreactions. Chem. Soc. Rev. 2013, 42 (20), $8122-$ 8133; (c) Crisenza, G. E. M.; Mazzarella, D.; Melchiorre, P., Synthetic Methods Driven by the Photoactivity of Electron Donor-Acceptor Complexes. J. Am. Chem. Soc. 2020, 142, 5461-5476.

22. (a) Goetz, F. J., Heterocyclic tautomerisms. I. An investigation of the 2-arylbenzothiazoline-2(benzylideneamino)thiophenol tautomerism. Part 1. J. Heterocycl. Chem. 1967, 4 (1), 80-84; (b) Goetz, F. J., Heterocyclic tautomerisms. III. An investigation of the 2-arylbenzothiazoline-2(benzylideneamino)thiophenol tautomerism. Part 3. J. Heterocycl. Chem. 1968, 5 (4), 509-512; (c) Mashraqui, S. H.; Kellogg, R. M., A ring expansion method for the preparation of 2,3-dihydro-1,4-benzothiazines from 2aryl-2,3-dihydrobenzothiazoles. Tetrahedron Lett. 1985, 26 (11), 1457-1460.

23. Aganda, K. C. C.; Kim, J.; Lee, A., Visible-light-mediated direct C3-arylation of 2H-indazoles enabled by an electron-donor-acceptor complex. Org. Biomol. Chem. 2019, 17 (45), 9698-9702. 